\title{
WAVE PROPAGATION IN VISCOELASTIC HALF-SPACE WITH MEMORY FUNCTIONS OF MITTAG-LEFFLER TYPE
}

\author{
Emilia Bazhlekova1 ${ }^{1}$, Sergey Pshenichnov ${ }^{2}$ \\ ${ }^{1}$ Institute of Mathematics and Informatics \\ Bulgarian Academy of Sciences \\ Acad. G. Bonchev Str., Bl. 8, Sofia - 1113, BULGARIA \\ ${ }^{2}$ Institute of Mechanics \\ Lomonosov Moscow State University \\ Michurinsky Prosp. 1, Moscow - 119192, RUSSIA
}

\begin{abstract}
The problem of one-dimensional non-stationary wave propagation in viscoelastic half space is studied. For the description of the hereditary properties of the viscoelastic medium, several examples of completely monotone relaxation kernels are considered. They are expressed in terms of functions of Mittag-Leffler type, including the recently introduced multinomial Prabhakar type function. Applying Laplace transform in time, some characteristics of the propagation function are discussed, such as non-negativity, monotonicity, propagation speed, presence/absence of wave front, and explicit integral representation of the solution is derived.
\end{abstract}

AMS Subject Classification: 33E12, 74D05, 74J05

Key Words: wave propagation; linear viscoelasticity; completely monotone function; multinomial Mittag-Leffler function; Laplace transform

\section{Introduction}

Analytical methods play an important role in the study of non-stationary wave processes in linear viscoelastic media $[2,18]$. To describe the hereditary properties of a viscoelastic medium, the stress and strain are related via a Volterra

Received: January 27, 2021

(C) 2021 Academic Publications

${ }^{\S}$ Correspondence author 
integral operator with a specific kernel, or, alternatively, via fractional calculus operators. For a comparative analysis we refer to [18, 27]. A historical survey of the early contributions to this topic can be found in [19]. As a generalization of the exponential relaxation kernel, characteristic for the Standard Linear Solid model, in his pioneering paper [24] Rabotnov proposed the so-called fractional exponential function. In fact, the Rabotnov function is a particular case of a completely monotone Mittag-Leffler type function.

In the present work we discuss further generalizations for the relaxation kernel, expressed in terms of functions of Mittag-Leffler type, including the recently introduced binomial Prabhakar function $[8,6]$. In particular cases we recover some known relaxation models, such as the fractional Zener model $[18,20]$, the distributed order fractional Zener model in the case of discrete distribution [1, 2], and the Havriliak-Negami relaxation model [9, 13]. The proposed in this work new relaxation kernel, which is expressed in terms of a completely monotone binomial Prabhakar type function, generalizes the above mentioned models.

The present study is a continuation of works $[16,17]$, where one-dimensional problems for wave propagation in viscoelastic solids are considered in the case when hereditary properties are characterized by two-parameter exponential relaxation kernel. We consider the problem of one-dimensional non-stationary wave propagation in viscoelastic half space, when initial conditions are set to zero and displacement is determined on the half space boundary. The solution of this problem is essential, as it allows obtaining solutions of more general, possibly multidimensional dynamic problems of linear viscoelasticity, by applying the subordination principle [22], Chapter 4. For the study of the problem and determination of the propagation and the Green functions Laplace transform in time is used. Explicit integral representation of the solution is derived. Some of the characteristics of the propagation function are discussed, such as nonnegativity, monotonicity, propagation speed, presence/absence of wave front.

The rest of the paper is organized as follows. Section 2 contains definitions and basic properties of the classical Mittag-Leffler type functions and their binomial versions. In Section 3 we set assumptions on the memory function, which ensure thermodynamic acceptability of the considered viscoelastic model. In Section 4 we discuss four specific examples of memory kernels of MittagLeffler type, satisfying the thermodynamic acceptability assumptions. Section 5 is devoted to the properties of the propagation function and deriving an explicit integral representation of the solution. 


\section{Preliminaries}

The Mittag-Leffler function is defined by the series [11]

$$
E_{\alpha, \beta}(z)=\sum_{k=0}^{\infty} \frac{z^{k}}{\Gamma(\beta+\alpha k)}, \quad z \in \mathbb{C}, \alpha, \beta \in \mathbb{R}, \alpha>0,
$$

where $\Gamma(\cdot)$ is the Gamma function. If $\beta=1$ the short notation $E_{\alpha}=E_{\alpha, 1}$ is usually used. The Mittag-Leffler function (1) is a generalization of the exponential function $\mathrm{e}^{z}$, which is recovered for $\alpha=\beta=1$.

The Prabhakar (or three-parameter Mittag-Leffler) function is defined as follows $[11,23]$

$$
E_{\alpha, \beta}^{\delta}(z)=\sum_{k=0}^{\infty} \frac{(\delta)_{k}}{k !} \frac{z^{k}}{\Gamma(\beta+\alpha k)}, \quad z \in \mathbb{C}, \alpha, \beta, \delta \in \mathbb{R}, \alpha>0,
$$

where $(\delta)_{k}$ denotes the Pochhammer symbol

$$
(\delta)_{k}=\frac{\Gamma(\delta+k)}{\Gamma(\delta)}=\delta(\delta+1) \ldots(\delta+k-1), \quad k \in \mathbb{N} ; \quad(\delta)_{0}=1
$$

The identity $(1)_{k}=k$ ! implies that for $\delta=1$ the Prabhakar function (2) reduces to the Mittag-Leffler function (1), i.e. $E_{\alpha, \beta}=E_{\alpha, \beta}^{1}$.

The following binomial Mittag-Leffler function of Prabhakar type has been recently introduced in [8]:

$$
E_{\left(\alpha_{1}, \alpha_{2}\right), \beta}^{\delta}\left(z_{1}, z_{2}\right)=\sum_{k=0}^{\infty} \sum_{l=0}^{\infty} \frac{(\delta)_{k+l}}{k ! l !} \frac{z_{1}^{k} z_{2}^{l}}{\Gamma\left(\beta+\alpha_{1} k+\alpha_{2} l\right)},
$$

where $z_{1}, z_{2} \in \mathbb{C}, \alpha_{1}, \alpha_{2}, \beta, \delta \in \mathbb{R}, \alpha_{1}>0, \alpha_{2}>0$. It satisfies the commutativity identity

$$
E_{\left(\alpha_{1}, \alpha_{2}\right), \beta}^{\delta}\left(z_{1}, z_{2}\right)=E_{\left(\alpha_{2}, \alpha_{1}\right), \beta}^{\delta}\left(z_{2}, z_{1}\right) .
$$

If one of the variables vanishes, function (3) reduces to the Prabhakar function of one variable $(2)$, i.e. $E_{\left(\alpha_{1}, \alpha_{2}\right), \beta}^{\delta}\left(z_{1}, 0\right)=E_{\alpha_{1}, \beta}^{\delta}\left(z_{1}\right)$.

The multinomial version of function (3) is studied in [6]. For other types of multi-index and multi-variable generalizations of the classical Mittag-Leffler function we refer to the recent surveys $[14,15,21]$ and the last edition of the monograph [11].

Let us denote the Laplace transform of a function $f(t)$ by $\widehat{f}$ or $\mathcal{L}\{f\}$, that is 


$$
\widehat{f}(s)=\mathcal{L}\{f(t)\}(s)=\int_{0}^{\infty} \mathrm{e}^{-s t} f(t) \mathrm{d} t .
$$

Of particular interest are the Prabhakar-type function

$$
\mathcal{E}_{\alpha, \beta}^{\delta}(t)=t^{\beta-1} E_{\alpha, \beta}^{\delta}\left(-\lambda t^{\alpha}\right)
$$

and its binomial generalization

$$
\mathcal{E}_{\left(\alpha_{1}, \alpha_{2}\right), \beta}^{\delta}(t)=t^{\beta-1} E_{\left(\alpha_{1}, \alpha_{2}\right), \beta}^{\delta}\left(-\lambda_{1} t^{\alpha_{1}},-\lambda_{2} t^{\alpha_{2}}\right),
$$

where $\lambda, \lambda_{1}, \lambda_{2} \in \mathbb{R}, t>0$. The series representation of the function $\mathcal{E}_{\left(\alpha_{1}, \alpha_{2}\right), \beta}^{\delta}(t)$ follows from (3):

$$
\mathcal{E}_{\left(\alpha_{1}, \alpha_{2}\right), \beta}^{\delta}(t)=\sum_{k=0}^{\infty} \sum_{l=0}^{\infty} \frac{(-1)^{k+l}(\delta)_{k+l}}{k ! l !} \frac{\lambda_{1}^{k} \lambda_{2}^{l} t^{\beta-1+\alpha_{1} k+\alpha_{2} l}}{\Gamma\left(\beta+\alpha_{1} k+\alpha_{2} l\right)} .
$$

The Laplace transform pair $\mathcal{L}\left\{\frac{t^{\beta-1}}{\Gamma(\beta)}\right\}=s^{-\beta}, \quad \beta>0$, yields the following identities for $\beta>0$ (for the first see e.g. [11], for the second see [6, 8])

$$
\mathcal{L}\left\{\mathcal{E}_{\alpha, \beta}^{\delta}(t)\right\}(s)=\frac{s^{-\beta}}{\left(1+\lambda s^{-\alpha}\right)^{\delta}}
$$

and

$$
\mathcal{L}\left\{\mathcal{E}_{\left(\alpha_{1}, \alpha_{2}\right), \beta}^{\delta}(t)\right\}(s)=\frac{s^{-\beta}}{\left(1+\lambda_{1} s^{-\alpha_{1}}+\lambda_{2} s^{-\alpha_{2}}\right)^{\delta}} .
$$

The Prabhakar type function $\mathcal{E}_{\alpha, \beta}^{\delta}(t)$ admits an asymptotic expansion for $t \rightarrow 0$ given by the first terms in the power series (2). For large $t$ it holds [10]

$$
\mathcal{E}_{\alpha, \beta}^{\delta}(t) \sim\left\{\begin{array}{l}
\lambda^{-\delta} \frac{t^{\beta-\alpha \delta-1}}{\Gamma(\beta-\alpha \delta)}, \alpha \delta \neq \beta, \\
-\delta \lambda^{-\delta-1} \frac{t^{-\alpha-1}}{\Gamma(-\alpha)}, \alpha \delta=\beta,
\end{array} \quad t \rightarrow+\infty .\right.
$$

Regarding the binomial Prabhakar type function (5) with $\alpha_{1}>\alpha_{2}$, the first terms in the power series (6) give the following asymptotic expansion for small $t$ :

$$
\mathcal{E}_{\left(\alpha_{1}, \alpha_{2}\right), \beta}^{\delta}(t) \sim \frac{t^{\beta-1}}{\Gamma(\beta)}-\delta \lambda_{2} \frac{t^{\beta+\alpha_{2}-1}}{\Gamma\left(\beta+\alpha_{2}\right)}, \quad t \rightarrow 0 .
$$


The leading terms in the asymptotic expansion for large $t$ are derived in [6] as follows:

$$
\mathcal{E}_{\left(\alpha_{1}, \alpha_{2}\right), \beta}^{\delta}(t) \sim\left\{\begin{array}{l}
\lambda_{1}^{-\delta} \frac{t^{\beta-\alpha_{1} \delta-1}}{\Gamma\left(\beta-\alpha_{1} \delta\right)}, \quad \alpha_{1} \delta \neq \beta, \\
-\delta \lambda_{1}^{-\delta-1} \lambda_{2} \frac{t^{-\alpha_{1}+\alpha_{2}-1}}{\Gamma\left(-\alpha_{1}+\alpha_{2}\right)}, \alpha_{1} \delta=\beta,
\end{array} \quad t \rightarrow+\infty .\right.
$$

In this work we will need also the following integration rule $[8,6]$

$$
\int_{0}^{t} \mathcal{E}_{\left(\alpha_{1}, \alpha_{2}\right), \beta}^{\delta}(\tau) \mathrm{d} \tau=\mathcal{E}_{\left(\alpha_{1}, \alpha_{2}\right), \beta+1}^{\delta}(t), \quad t>0
$$

which can be established directly from the series definition (5), or by applying Laplace transform and using (8). Identity (12) generalizes a property for the standard Prabhakar function, which can be derived from (12) replacing $\left(\alpha_{1}, \alpha_{2}\right)$ with $\alpha$.

An essential feature of the functions of Mittag-Leffler type is related to the property of complete monotonicity. A function $f:(0, \infty) \rightarrow \mathbb{R}$ is said to be completely monotone function $(\mathcal{C M F})$ if it is of class $C^{\infty}$ and

$$
(-1)^{n} f^{(n)}(t) \geq 0, \quad t>0, n=0,1,2, \ldots
$$

The characterization of the class $\mathcal{C M F}$ is given by the Bernstein's theorem: a function is completely monotone if and only if it can be represented as the Laplace transform of a non-negative (generalized) function. The class $\mathcal{C M F}$ is closed under pointwise addition, multiplication and convergence [28, 22].

Let $\lambda>0$. The Prabhakar type function $\mathcal{E}_{\alpha, \beta}^{\delta}(t)$ given in (4) is completely monotone if the parameters satisfy the conditions [10]

$$
0<\alpha \leq 1,0<\alpha \delta \leq \beta \leq 1
$$

In particular, the function of Mittag-Leffler type $\mathcal{E}_{\alpha, \beta}(t) \in \mathcal{C M F}$ provided $0<\alpha \leq \beta \leq 1$.

Let $\lambda_{1}, \lambda_{2}>0$. It is proven in [6] that the binomial Prabhakar type function $\mathcal{E}_{\left(\alpha_{1}, \alpha_{2}\right), \beta}^{\delta}(t)$ given in (5) is completely monotone provided

$$
0<\alpha_{j} \leq 1,0<\alpha_{j} \delta \leq \beta \leq 1, \quad j=1,2 .
$$




\section{Admissible memory functions}

The following one-dimensional problem, governing wave propagation in viscoelastic half-space, is considered in [16]

$$
\begin{aligned}
& \ddot{u}=u^{\prime \prime}-m * u^{\prime \prime}, \quad x, t>0, \\
& \left.u\right|_{x=0}=u_{0}(t), \quad|u|<\infty \text { as } x \rightarrow \infty, \quad t>0, \\
& \left.u\right|_{t=0}=\left.\dot{u}\right|_{t=0}=0, \quad x>0,
\end{aligned}
$$

where $u=u(x, t)$ is displacement, $x$ and $t$ are dimensionless spatial and temporal variables, primes and dots correspond to the dimensionless space and time derivatives, respectively, $m=m(t)$ is a given memory function, which will be specified later, and $*$ denotes the Laplace convolution with respect to the variable $t$ :

$$
(f * g)(t)=\int_{0}^{t} f(t-\tau) g(\tau) d \tau .
$$

Problem (16)-(18) is conveniently treated using Laplace transform. Let us denote by $\widehat{u}(x, s)$ the Laplace transform of the function $u(x, t)$ with respect to $t$, i.e.

$$
\widehat{u}(x, s)=\int_{0}^{\infty} \mathrm{e}^{-s t} u(x, t) \mathrm{d} t .
$$

By applying Laplace transform to equation (16) and taking into account the boundary conditions (17) and initial conditions (18), the following ODE for $\widehat{u}(x, s)$ is obtained

$$
s^{2}(1-\widehat{m}(s))^{-1} \widehat{u}(x, s)=\widehat{u}^{\prime \prime}(x, s), \widehat{u}(0, s)=\widehat{u}_{0}(s),
$$

where $\widehat{u}(x, s)$ is bounded for $x \rightarrow \infty$. Solving problem (19) with $s$ considered as a parameter, it follows

$$
\widehat{u}(x, s)=\widehat{u}_{0}(s) \mathrm{e}^{-\mu(s) x},
$$

where

$$
\mu(s)=s(1-\widehat{m}(s))^{-1 / 2} .
$$

In [16] an exponential memory kernel is considered: $m(t)=C \mathrm{e}^{-\lambda t}$. Our aim in this work is to expand the class of memory functions $m(t)$ in a physically acceptable way, by involving functions of Mittag-Leffler type. To this end we use the representation of the wave equation in terms of the so-called relaxation modulus $G(t)$, which is a characteristic function for a viscoelastic medium. The 
second law of thermodynamics, stating that the total entropy can only increase over time for an isolated system, implies that $G(t)$ should be a non-negative and non-increasing function for $t>0$. In this work a solid-like viscoelastic medium is considered, i.e. the creep is supposed finite as $t \rightarrow \infty$, which corresponds to the assumption $G(+\infty)>0$, i.e. $G(t)>0$ for all $t \geq 0$. For more details on the theory of linear viscoelasticity we refer to $[18,20]$.

The equation for wave propagation in linear viscoelastic media is written in the following form

$$
\dot{u}=G * u^{\prime \prime}, \quad x, t>0,
$$

where, as above, primes and dots correspond to the dimensionless space and time derivatives, respectively, and $G(t)$ is the relaxation modulus. Integration of both sides of equation (16), by taking into account the initial conditions (18), leads to an equation of the form (22), where

$$
G(t)=1-\int_{0}^{t} m(\tau) \mathrm{d} \tau, \quad t \geq 0 .
$$

The obtained relation (23) between the relaxation modulus $G(t)$ and the memory function $m(t)$ can be also written in a differentiated form as

$$
m(t)=-\frac{\mathrm{d} G}{\mathrm{~d} t}, \quad t \geq 0 .
$$

This means that the function $m(t)$ is a measure of the rate of relaxation. Based on the properties of the relaxation modulus $G(t)$ we derive next restrictions on the memory function $m(t)$.

First, since $G(t)$ should be a non-increasing function for $t>0$, from (24) we deduce that $m(t) \geq 0$ for any $t \geq 0$. Next, the relation (23) between the functions $G(t)$ and $m(t)$ implies that the property $G(t)>0$ is equivalent to $1-\int_{0}^{t} m(\tau) \mathrm{d} \tau>0$ for any $t \geq 0$, which is ensured by assuming that $m(t)$ is integrable on $(0, \infty)$ and

$$
\int_{0}^{\infty} m(t) \mathrm{d} t<1 .
$$

Assumption $(25)$ can also be written as $\widehat{m}(0)<1$.

An important class of thermodynamically admissible relaxation moduli is the class of completely monotone relaxation functions $G(t)$, c.f. $[13,18]$. Relation (24) implies that in this case the function $m(t)$ should also be completely monotone.

In the rest of this work we make the following assumptions on the memory function: 
(A) $m(t) \in \mathcal{C M F}, m(t) \in L^{1}(0, \infty)$ and (25) is satisfied.

Let us briefly discuss some implications of assumptions (A).

Let $s \in \mathbb{R}, s \geq 0$. We note that since $m(t) \geq 0$, then $\widehat{m}(s) \in \mathcal{C M F}$, in particular, $\widehat{m}(s)$ is a nonnegative and nonincreasing function in $s \geq 0$. Therefore, (25) implies that $\widehat{m}(s) \leq \widehat{m}(0)<1$ for any $s \geq 0$. Moreover, according to [12], Theorem 2.6, the complete monotonicity of $m(t)$ implies that

$$
\lim _{s \rightarrow+\infty} \widehat{m}(s)=0
$$

and that the function $\widehat{m}(s)$ admits an analytic extension to the whole complex plane cut along the negative real axis.

Assumptions (A) imply for the relaxation modulus $G(t)$ that it is a locally integrable function, such that $G(t) \in \mathcal{C M F}$. Indeed, (23) implies that $G(t)$ is a continuous function and $G(0)=1$, therefore, it is locally integrable. Since $m(t) \in \mathcal{C} \mathcal{M F}$ then (24) implies that the derivatives of $G(t)$ of all orders satisfy the inequalities (13). To establish complete monotonicity of $G(t)$ it remains to check that $G(t) \geq 0$ for all $t \geq 0$. Since $\dot{G}(t) \leq 0$, the function $G(t)$ is nonincreasing. Moreover, (23) together with inequality (25) implies $G(+\infty)>0$. Therefore, $G(t)>0$ for all $t \geq 0$. In this way, we established that assumptions (A) imply the complete monotonicity of the relaxation modulus $G(t)$ and $G(+\infty)>0$, which corresponds to solid-like viscoelastic behavior.

The relation (23) between the relaxation modulus $G(t)$ and the function $m(t)$ implies

$$
\widehat{G}(s)=\frac{1-\widehat{m}(s)}{s},
$$

which together with (21) yields

$$
\mu(s)=\left(\frac{s}{\widehat{G}(s)}\right)^{1 / 2} .
$$

Let us consider briefly the Green function of problem (16)-(18), which corresponds to $u_{0}(t)=\delta(t)$, the Dirac delta function. The Laplace transform of the Green function is obtained by setting $\widehat{u}_{0}(s)=\widehat{\delta}(s)=1$ in (20). Since under assumptions (A) the function $G(t)$ is locally integrable function and $G(t) \in \mathcal{C M F}$, applying the technique of Bernstein functions, it follows (for details see e.g. [5]) that $\mathrm{e}^{-\mu(s) x} \in \mathcal{C} \mathcal{M F}$ in the variable $s \geq 0$, when $x>0$ is considered as a parameter. Therefore, by the Bernstein's theorem, the Green function is nonnegative. This is naturally expected in a physically meaningful model. 


\section{Relaxation of Mittag-Leffler type}

In this section we consider several examples of relaxation functions $m(t)$, which satisfy assumptions (A). They are generalizations of the classical case $m(t)=$ $C \mathrm{e}^{-\lambda t}, 0<C<\lambda$. In this classical case conditions (A) are easily verified. Relation (23) yields

$$
G(t)=\left(1-\frac{C}{\lambda}\right)+\frac{C}{\lambda} \mathrm{e}^{-\lambda t}, \quad t \geq 0,
$$

and we recognize the classical Zener (or Standard Linear Solid) relaxation model, see e.g. [18, 20].

\subsection{Memory kernel of Mittag-Leffler type}

Let $0<\alpha \leq 1$ and consider the following memory kernel in terms of MittagLeffler function

$$
m(t)=C t^{\alpha-1} E_{\alpha, \alpha}\left(-\lambda t^{\alpha}\right), \widehat{m}(s)=\frac{C}{s^{\alpha}+\lambda}, \quad 0<C<\lambda .
$$

Kernel (29) reduces to the above exponential kernel for $\alpha=1$. The parameters satisfy conditions (14) with $\alpha=\beta$ and $\delta=1$. Therefore, $m(t) \in \mathcal{C M F}$. Asymptotic expansions in the next subsection (35) and (36) with $\delta=1$ give the behavior of $m(t)$ and show that it is integrable function on $(0, \infty)$. Assumption $0<C<\lambda$ implies $\widehat{m}(0)<1$. Hence, properties (A) are satisfied. To find the relaxation modulus $G(t)$ we can use identity (23), which together with (12) implies

$$
G(t)=1-C t^{\alpha} E_{\alpha, \alpha+1}\left(-\lambda t^{\alpha}\right) .
$$

For an alternative representation, let us find the Laplace transforms of the relaxation modulus $G(t)$ by applying relation (27). Setting

$$
a=\lambda^{-1}, \quad b=(\lambda-C)^{-1},
$$

it follows in the case of kernel (29)

$$
\widehat{G}(s)=\frac{a}{b s} \frac{1+b s^{\alpha}}{1+a s^{\alpha}}, \quad a<b,
$$

which suggests that this is the fractional Zener relaxation model, see e.g. [3, 18]. After inversion of the LT in (32) we derive by the use of identity (7) with $\beta=1$ and $\delta=1$ the following, alternative to (30), representation

$$
G(t)=\left(1-\frac{C}{\lambda}\right)+\frac{C}{\lambda} E_{\alpha}\left(-\lambda t^{\alpha}\right) .
$$


We see from (33) that $G(t) \in \mathcal{C M F}$. Moreover, taking into account the asymptotic expansion (9) of the Mittag-Leffler functions appearing in (30) or (33) it follows $G(+\infty)=1-C / \lambda>0$.

\subsection{Memory kernel of Prabhakar type}

Let $0<\alpha \leq 1$ and $0<\delta \leq 1$. As a further generalization, consider the Prabhakar type memory kernel

$$
m(t)=C t^{\alpha \delta-1} E_{\alpha, \alpha \delta}^{\delta}\left(-\lambda t^{\alpha}\right), \widehat{m}(s)=\frac{C}{\left(s^{\alpha}+\lambda\right)^{\delta}}, 0<C<\lambda^{\delta},
$$

which for $\delta=1$ reduces to (29). The asymptotic behaviors of the memory function (34) for short and long times are derived by the use of (2) and (9):

$$
\begin{aligned}
& m(t) \sim C \frac{t^{\alpha \delta-1}}{\Gamma(\alpha \delta)}, \quad t \rightarrow 0, \\
& m(t) \sim-C \delta \lambda^{-\delta-1} \frac{t^{-\alpha-1}}{\Gamma(-\alpha)}, \quad t \rightarrow+\infty .
\end{aligned}
$$

Therefore, $m(t)$ is integrable function on $(0, \infty)$. The parameters in (34) satisfy conditions $(14)$ for complete monotonicity and $\widehat{m}(0)<1$. Hence, $m(t)$ satisfies properties (A). Applying identity (27) we obtain for the relaxation modulus $G(t)$ in the case of kernel (34)

$$
\widehat{G}(s)=\frac{1}{s}\left(1-\frac{C}{\left(s^{\alpha}+\lambda\right)^{\delta}}\right)=\frac{1}{s}\left(1-\frac{d}{\left(a s^{\alpha}+1\right)^{\delta}}\right),
$$

where $a=\lambda^{-1}$ and $d=C / \lambda^{\delta}<1$. Therefore, we recognize from (37) the relaxation modulus of the Havriliak-Negami relaxation model, see e.g. [13, 9]. Relation (23) and the integration rule (12) imply

$$
G(t)=1-C t^{\alpha \delta} E_{\alpha, \alpha \delta+1}^{\delta}\left(-\lambda t^{\alpha}\right) .
$$

The asymptotic expansion (9) then implies $G(+\infty)=1-C / \lambda^{\delta}>0$.

In the particular case $\alpha=1$ the Prabhakar kernel (34) reduces to the tempered power-law memory kernel

$$
m(t)=C \frac{t^{\delta-1}}{\Gamma(\delta)} \mathrm{e}^{-\lambda t}, \quad \widehat{m}(s)=\frac{C}{(s+\lambda)^{\delta}}, \quad 0<C<\lambda^{\delta} .
$$

Therefore, the model with memory function (39) appears to be a particular case of the Havriliak-Negami relaxation model. Plugging $\alpha=1$ in (38) yields the following completely monotone relaxation modulus in the case of memory kernel (39):

$$
G(t)=1-C t^{\delta} E_{1, \delta+1}^{\delta}(-\lambda t) .
$$




\subsection{Memory kernel of binomial Mittag-Leffler type}

Let $0<\alpha_{0}<\alpha \leq 1, \lambda>0, \lambda_{0}>0$, and consider the binomial Mittag-Leffler memory function

$$
m(t)=C t^{\alpha-1} E_{\left(\alpha, \alpha_{0}\right), \alpha}\left(-\lambda t^{\alpha},-\lambda_{0} t^{\alpha_{0}}\right), \quad 0<C<\lambda .
$$

Let us note that for $\lambda_{0}=0$ kernel (40) reduces to (29). The parameters satisfy conditions (15), therefore $m(t) \in \mathcal{C} \mathcal{M F}$. Asymptotic expansions (43) and (44) with $\delta=1$ give the behavior of kernel (40) and show that it is integrable function on $(0, \infty)$. Laplace transform pair (8) yields

$$
\widehat{m}(s)=\frac{C}{s^{\alpha}+\lambda_{0} s^{\alpha-\alpha_{0}}+\lambda} .
$$

Therefore $\widehat{m}(0)<1$ and properties (A) are fulfilled.

In the case of the binomial Mittag-Leffler memory kernel (40) the relaxation modulus, obtained inserting (41) in (27) equals

$$
\widehat{G}(s)=\frac{a}{b s} \frac{1+b_{1} s^{\alpha-\alpha_{0}}+b s^{\alpha}}{1+a_{1} s^{\alpha-\alpha_{0}}+a s^{\alpha}}
$$

where the coefficients $a, b$ are defined in (31) and $a_{1}=\lambda_{0} a, b_{1}=\lambda_{0} b$. Therefore, we recognize a multi-term fractional Zener model (distributed-order fractional Zener model in the case of discrete distribution) which relaxation modulus is completely monotone, see $[1,7]$. Rheological models of this type and related simple mechanical systems are discussed also in $[4,25,27]$.

The explicit form of $G(t)$ derived from (40), (23), and (12), is

$$
G(t)=1-C t^{\alpha} E_{\left(\alpha, \alpha_{0}\right), \alpha+1}\left(-\lambda t^{\alpha},-\lambda_{0} t^{\alpha_{0}}\right)
$$

and the asymptotic expansion (11) yields $G(+\infty)=1-C / \lambda>0$.

\subsection{Memory kernel of binomial Prabhakar type}

Let $0<\alpha_{0}<\alpha \leq 1,0<\delta \leq 1$, and $\lambda, \lambda_{0}>0$. Consider as a last generalization the binomial memory function of Prabhakar type

$$
m(t)=C t^{\alpha \delta-1} E_{\left(\alpha, \alpha_{0}\right), \alpha \delta}^{\delta}\left(-\lambda t^{\alpha},-\lambda_{0} t^{\alpha_{0}}\right), \quad 0<C<\lambda^{\delta} .
$$

Let us note that kernel (42) reduces to (34) if $\lambda_{0}=0$ and to (40) if $\delta=1$. Again, the parameters of the binomial Prabhakar kernel satisfy conditions (15). Therefore $m(t) \in \mathcal{C} \mathcal{M F}$. 
For completeness, we give next the asymptotic behavior of the memory functions (42), derived by the use of (10) and (11):

$$
\begin{aligned}
& m(t) \sim C \frac{t^{\alpha \delta-1}}{\Gamma(\alpha \delta)}, \quad t \rightarrow 0 \\
& m(t) \sim-C \delta \lambda^{-\delta-1} \lambda_{0} \frac{t^{-\alpha+\alpha_{0}-1}}{\Gamma\left(-\alpha+\alpha_{0}\right)}, \quad t \rightarrow+\infty
\end{aligned}
$$

Therefore, $m(t)$ is integrable function on $(0, \infty)$.

Laplace transform pair (8) yields for the kernel (42)

$$
\widehat{m}(s)=\frac{C}{\left(s^{\alpha}+\lambda_{0} s^{\alpha-\alpha_{0}}+\lambda\right)^{\delta}},
$$

which implies $\widehat{m}(0)<1$. Therefore, properties $(\mathbf{A})$ are satisfied for the kernel (42).

The corresponding relaxation modulus $G(t)$, derived from (42), (23), and $(12)$, is

$$
G(t)=1-C t^{\alpha \delta} E_{\left(\alpha, \alpha_{0}\right), \alpha \delta+1}^{\delta}\left(-\lambda t^{\alpha},-\lambda_{0} t^{\alpha_{0}}\right) .
$$

The function $G(t)$ is completely monotone, which follows from the fact that $m(t)$ satisfies properties (A). The asymptotic expansions of $G(t)$ for $t \rightarrow 0$ and $t \rightarrow+\infty$ are obtained from (45) by applying (10) and (11), respectively:

$$
\begin{aligned}
G(t) & \sim 1-C \frac{t^{\alpha \delta}}{\Gamma(\alpha \delta+1)}+C \delta \lambda_{0} \frac{t^{\alpha \delta+\alpha_{0}}}{\Gamma\left(\alpha \delta+\alpha_{0}+1\right)}, \quad t \rightarrow 0, \\
G(t) & \sim 1-\frac{C}{\lambda^{\delta}}+C \delta \lambda^{-\delta-1} \lambda_{0} \frac{t^{-\alpha+\alpha_{0}-1}}{\Gamma\left(-\alpha+\alpha_{0}\right)}, \quad t \rightarrow+\infty .
\end{aligned}
$$

In particular, $G(0)=1, G(+\infty)=1-C / \lambda^{\delta}$, i.e. the function $G(t)$ is monotonically decreasing in $(0,+\infty)$ from $G(0)=1$ to $G(+\infty) \in(0,1)$.

The Laplace transform of the relaxation modulus in the case of the binomial Prabhakar memory kernel (42) can be obtained taking into account (27) as follows

$$
\widehat{G}(s)=\frac{1}{s}\left(1-\frac{d}{\left(a s^{\alpha}+a_{1} s^{\alpha-\alpha_{0}}+1\right)^{\delta}}\right),
$$

where $a=\lambda^{-1}, a_{1}=\lambda_{0} \lambda^{-1}$, and $d=C / \lambda^{\delta}<1$. Therefore, this model is a generalization of the Havriliak-Negami relaxation model, to which it reduces when $\lambda_{0}=0\left(a_{1}=0\right)$, as well as a further generalization of the Zener model considered in the previous Subsection 4.3. 
It is worth noting that in Subsections 4.3 and 4.4 the multinomial variants of Mittag-Leffler and Prabhakar type functions can be considered by an analogous argument and applying complete monotonicity results established in [6].

\section{Propagation function}

The solution of problem (16)-(18) with boundary condition $u_{0}(t)=H(t)$, where $H(t)$ is the Heaviside unit step function, is referred to as propagation function. Let us denote the propagation function by $w(x, t)$. Then from (20), and taking into account that $\widehat{H}(s)=1 / s$, it follows

$$
\widehat{w}(x, s)=\frac{1}{s} \mathrm{e}^{-x \mu(s)} .
$$

Let us first discuss the behavior of the propagation function, based on its Laplace transform (46) for $s \in \mathbb{R}, s \geq 0$. As mentioned before, $\mathrm{e}^{-x \mu(s)} \in \mathcal{C M F}$ in $s \geq 0(x>0$ is considered as a parameter $)$. Since $1 / s \in \mathcal{C M \mathcal { F }}$, then $\widehat{w}(x, s) \in \mathcal{C} \mathcal{M F}$ as a function of $s$, being a product of two completely monotone functions. Then Bernstein's theorem implies $w(x, t) \geq 0$. Taking into account the initial condition $w(x, 0)=0$, (46) implies

$$
\mathcal{L}\{\dot{w}\}(x, s)=s \widehat{w}(x, s)-w(x, 0)=\mathrm{e}^{-x \mu(s)} .
$$

Therefore, $\mathcal{L}\{\dot{w}\}(x, s) \in \mathcal{C M F}$ in $s \geq 0$ and Bernstein's theorem implies $\dot{w}(x, t) \geq 0$, thus, the function $w(x, t)$ is nondecreasing in $t$. To derive the limit for large times we can apply the final value theorems for the Laplace transform. The Laplace transform $\widehat{w}(x, s)$ has no poles in the complex plane except a single pole at $s=0$. Therefore,

$$
\lim _{t \rightarrow+\infty} w(x, t)=\lim _{s \rightarrow 0} s \widehat{w}(x, s)=\lim _{s \rightarrow 0} \mathrm{e}^{-x \mu(s)}=1 .
$$

Here we have used that $\mu(0)=0$, following from $(21)$ and $\widehat{m}(0)<1$.

Characteristic for the behavior of the solution is the propagation speed of a disturbance ([18, Chapter 4], [22, Chapter 5])

$$
c=\lim _{s \rightarrow+\infty} \frac{s}{\mu(s)} .
$$

In the case of finite propagation speed $c$, a jump discontinuity at the planar surface $x=c t$ exists if [22, Section 5.4]

$$
\omega=\lim _{s \rightarrow+\infty}(\mu(s)-s / c)<\infty .
$$


Let us calculate the quantities $c$ and $\omega$ for the considered models. According to assumptions (A), $\widehat{m}(s) \rightarrow 0$ as $s \rightarrow+\infty$, see (26). Therefore, inserting (21) in (48) and (49) implies

$$
c=1 ; \quad \omega=\lim _{s \rightarrow+\infty} s\left((1-\widehat{m}(s))^{-1 / 2}-1\right)=\lim _{s \rightarrow+\infty} \frac{s \widehat{m}(s)}{2},
$$

where for the derivation of $\omega$ the asymptotic expansion $(1+z)^{b} \approx 1+b z$ for $|z|<1$ is used. By the initial value theorem for the Laplace transform, (50) implies $\omega=m(0) / 2$.

Therefore, in all models with memory function satisfying conditions (A) the propagation speed of a disturbance is $c=1$. This implies that the propagation function $w(x, t)$ vanishes for $t<x$.

Let us further discuss the behavior of $w(x, t)$ at the wave front $x=t$, which depends on the value of $m(0)$. The propagation function in the classical case $m(t)=C \mathrm{e}^{-\lambda t}, 0<C<\lambda$, studied in [16], exhibits a jump discontinuity at $x=t$, since $m(0)=C$ is finite. The memory functions considered in the previous section provide as well examples, in which the wave front at $x=t$ is smooth. Such are the models (29) and (40) with $\alpha<1$ and models (34) and (42) with $\alpha \delta<1$. For all these models $m(0)$ is infinite. On the other hand, model (40) with $\alpha=1$ and $\alpha_{0}<1$ provides an example of a memory kernel, different from the exponential, for which the propagation function $w(x, t)$ admits a jump discontinuity at $x=t$. Indeed, in this case (40) with $\alpha=1$ implies $m(0)=$ $C<\infty$.

Next we find an explicit integral expression for the propagation function by inverting the Laplace transform in (46). Applying the Bromwich integral inversion formula to (46) yields:

$$
w(x, t)=\frac{1}{2 \pi \mathrm{i}} \int_{\gamma-\mathrm{i} \infty}^{\gamma+\mathrm{i} \infty} \mathrm{e}^{s t} \widehat{w}(x, s) \mathrm{d} s=\frac{1}{2 \pi \mathrm{i}} \int_{\gamma-\mathrm{i} \infty}^{\gamma+\mathrm{i} \infty} \mathrm{e}^{s t-x \mu(s)} \frac{\mathrm{d} s}{s}, \quad \gamma>0 .
$$

In what follows, for multivalued functions in $\mathbb{C}$ such as $s^{\alpha}$ we consider always the principal branch. Due to the assumptions (A), we established that the relaxation modulus $G(t)$ is locally integrable and completely monotone. Then, representation (28) implies that $\mu(s)$ is a complete Bernstein function (for the proof we refer to [5], definition of this class and further details can be found in [28]). Therefore, $\mu(s)$ admits an analytic extension to $\mathbb{C} \backslash(-\infty, 0]$, such that $|\arg \mu(s)| \leq|\arg s|$ and $\mu\left(s^{*}\right)=(\mu(s))^{*}$, where $*$ denotes the complex conjugate. Applying the Cauchy's theorem, the integration on the Bromwich path $(\gamma-\mathrm{i} \infty, \gamma+\mathrm{i} \infty)$ can be replaced by integration on the contour $D \cup D_{0}$, where

$$
D=\{s=\mathrm{i} r, r \in(-\infty,-\varepsilon) \cup(\varepsilon, \infty)\},
$$




$$
D_{0}=\left\{s=\varepsilon e^{\mathrm{i} \theta}, \theta \in[-\pi / 2, \pi / 2]\right\} .
$$

Indeed, the integrals on the $\operatorname{arcs} D_{R}^{-}$and $D_{R}^{+}$, where

$$
D_{R}^{ \pm}=\{|s|=R, \Re s \in[0, \gamma], \pm \Im s>0\},
$$

vanish for $R \rightarrow \infty$. This follows from the fact that $\Re \mu(s) \geq 0$ for $\Re s \geq 0$ and therefore

$$
\left.\left|\frac{1}{s} \exp (-x \mu(s))\right| \leq \frac{1}{R} \exp (-x \Re \mu(s))\right) \leq \frac{1}{R}, \quad s \in D_{R}^{ \pm} .
$$

The integral on the semi-circular contour $D_{0}$ equals $1 / 2$ when $\varepsilon \rightarrow 0$. This can be obtained by direct check using that

$$
\lim _{s \rightarrow 0} s\left(\frac{1}{s} \exp (s t-x \mu(s))\right)=1 .
$$

Integration on the contour $D$ yields after letting $\varepsilon \rightarrow 0$

$$
\frac{1}{2 \pi \mathrm{i}} \int_{D} \frac{1}{s} \exp (s t-x \mu(s)) \mathrm{d} s=\frac{1}{\pi} \int_{0}^{\infty} \frac{1}{r} \Im \exp (\mathrm{i} r t-x \mu(\mathrm{i} r)) \mathrm{d} r .
$$

Applying the formula for real and imaginary parts of the square root of a complex number we obtain the following result for $x, t>0$

$$
w(x, t)=\frac{1}{2}+\frac{1}{\pi} \int_{0}^{\infty} \exp \left(-x K^{+}(r)\right) \sin \left(r t-x K^{-}(r)\right) \frac{\mathrm{d} r}{r},
$$

where

$$
K^{ \pm}(r)=\frac{r}{\sqrt{2}}\left(\left(A^{2}(r)+B^{2}(r)\right)^{1 / 2} \mp A(r)\right)^{1 / 2}
$$

with

$$
A(r)=\Re\left\{(1-\widehat{m}(\mathrm{i} r))^{-1}\right\}, \quad B(r)=\Im\left\{(1-\widehat{m}(\mathrm{i} r))^{-1}\right\} .
$$

For the Green function $\mathcal{G}(x, t)$ it holds $\widehat{\mathcal{G}}(x, s)=\mathrm{e}^{-x \mu(s)}$. Taking into account $(47)$, it follows by the uniqueness of the Laplace transform that $\mathcal{G}(x, t)=$ $\dot{w}(x, t)$ and the following integral representation for the Green function is obtained by time differentiation under the integral sign in (52)

$$
\mathcal{G}(x, t)=\frac{1}{\pi} \int_{0}^{\infty} \exp \left(-x K^{+}(r)\right) \cos \left(r t-x K^{-}(r)\right) \mathrm{d} r, \quad x, t>0,
$$

where the functions $K^{ \pm}(r)$ are defined in (53).

The derived integral representations for the solutions can be further used for numerical computation and visualization. 


\section{Acknowledgements}

This study was performed within the bilateral project funded by the Bulgarian National Science Fund, Project number KP-06-Russia/5 from 11.12.2020 and by the Russian Foundation for Basic Research, Project number 20-58-18002.

\section{References}

[1] T. Atanacković, S. Konjik, L. Oparnica, D. Zorica, Thermodynamical restrictions and wave propagation for a class of fractional order viscoelastic rods, Abstr. Appl. Anal., 2011 (2011), Art. \# 975694-1-32.

[2] T. Atanacković, S. Pilipović, B. Stanković, D. Zorica, Fractional Calculus with Applications in Mechanics: Vibrations and Diffusion Processes, John Wiley \& Sons, London (2014).

[3] T. Atanacković, S. Pilipović, D. Seleši, Wave propagation dynamics in a fractional Zener model with stochastic excitation, Fract. Calc. Appl. Anal., 23, No 6 (2020), 1570-1604; DOI:10.1515/fca-2020-0079.

[4] R.L. Bagley, P.J. Torvik, On the fractional calculus model of viscoelastic behavior. J. Rheol., 30 (1986), 137-148.

[5] E. Bazhlekova, Subordination in a class of generalized time-fractional diffusion-wave equations, Fract. Calc. Appl. Anal., 21, No 4 (2018), 869900; DOI:10.1515/fca-2018-0048.

[6] E. Bazhlekova, Completely monotone multinomial Mittag-Leffler type functions and diffusion equations with multiple time-derivatives, Fract. Calc. Appl. Anal., 24, No 1 (2021), 88-111; DOI:10.1515/fca-2021-0005.

[7] E. Bazhlekova, I. Bazhlekov, Complete monotonicity of the relaxation moduli of distributed-order fractional Zener model, AIP Conf. Proc., 2048 (2018), Art. \# 050008.

[8] A. Fernandez, C. Kürt, M. Özarslan, A naturally emerging bivariate Mittag-Leffler function and associated fractional calculus operators, Comp. Appl. Math., 39 (2020), Art. \# 200.

[9] R. Garrappa, F. Mainardi, G. Maione, Models of dielectric relaxation based on completely monotone functions, Fract. Calc. Appl. Anal., 19, No 5 (2016), 1105-1160; DOI:10.1515/fca-2016-0060. 
[10] A. Giusti, I. Colombaro, R. Garra, R. Garrappa, F. Polito, M. Popolizio, F. Mainardi, A practical guide to Prabhakar fractional calculus, Fract. Calc. Appl. Anal., 23, No 1 (2020), 9-54; DOI:10.1515/fca-2020-0002.

[11] R. Gorenflo, A. Kilbas, F. Mainardi, S. Rogosin, Mittag-Leffler Functions, Related Topics and Applications, 2nd Ed., Springer, Berlin-Heidelberg (2020).

[12] G. Gripenberg, S.O. Londen, O.J. Staffans, Volterra Integral and Functional Equations, Cambridge University Press, Cambridge (1990).

[13] A. Hanyga, Wave propagation in linear viscoelastic media with completely monotonic relaxation moduli, Wave Motion, 50 (2013), 909-928.

[14] V. Kiryakova, Unified approach to fractional calculus images of special functions - a survey, Mathematics, 8, No 12 (2020), Art. \# 2260; DOI:10.3390/math8122260.

[15] V. Kiryakova, A guide to special functions in fractional calculus, Mathematics, 9, No 1 (2021), Art. \# 106; DOI:10.3390/math9010106.

[16] E.A. Korovaytseva, S.G. Pshenichnov, D.V. Tarlakovskii, Propagation of one-dimensional non-stationary waves in viscoelastic half space, Lobachevskii Journal of Mathematics, 38 (2017), 827-832.

[17] E.A. Korovaytseva, S.G. Pshenichnov, D.V. Tarlakovskii, Analytical solution of non-stationary waves propagation in viscoelastic layer problem, Lobachevskii J. of Mathematics, 40 (2019), 2084-2089.

[18] F. Mainardi, Fractional Calculus and Waves in Linear Viscoelasticity, Imperial College Press, London (2010).

[19] F. Mainardi, An historical perspective on fractional calculus in linear viscoelasticity, Fract. Calc. Appl. Anal., 15, No 4 (2012), 712-717; DOI:10.2478/s13540-012-0048-6.

[20] F. Mainardi, G. Spada, Creep, relaxation and viscosity properties for basic fractional models in rheology, Eur. Phys. J. Special Topics, 193 (2011), $133-160$.

[21] J. Paneva-Konovska, V. Kiryakova, On the multi-index Mittag-Leffler functions and their Mellin transforms. Int. J. Appl. Math. 33, No 4 (2020), 549-571; DOI:10.12732/ijam.v33i4.1. 
[22] J. Prüss, Evolutionary Integral Equations and Applications, Birkhäuser, Basel-Boston-Berlin (1993).

[23] T.R. Prabhakar, A singular integral equation with a generalized Mittag-Leffler function in the kernel, Yokohama Math. J., 19, No 1 (1971), 7-15.

[24] Yu.N. Rabotnov, Equilibrium of an elastic medium with after effect, Prikl. Matem. i Mekh. (PMM), 12, No 1 (1948), 81-91 (in Russian).

[25] Yu.A. Rossikhin, M.V. Shitikova, Analysis of the viscoelastic rod dynamics via models involving fractional derivatives or operators of two different orders, Shock Vib. Digest 36, No 1 (2004), 3-26.

[26] Yu.A. Rossikhin, M.V. Shitikova, Comparative analysis of viscoelastic models involving fractional derivatives of different orders, Fract. Calc. Appl. Anal., 10, No 2 (2007), 111-121.

[27] Yu.A. Rossikhin, M.V. Shitikova, Application of fractional calculus for dynamic problems of solid mechanics: Novel trends and recent results. Appl. Mech. Rev. 63 (2010), Art. \# 010801-1-25.

[28] R. Schilling, R. Song, Z. Vondraček, Bernstein Functions: Theory and Applications, De Gruyter, Berlin (2010). 